\title{
Liquid friction on charged surfaces: from hydrodynamic slippage to electrokinetics
}

\author{
Laurent Joly ${ }^{1}$ ه Christophe Ybert ${ }^{1}$, Emmanuel Trizac ${ }^{2}$, and Lydéric Bocquet ${ }^{1}$ \\ 1 Laboratoire P.M.C.N., UMR CNRS 5586, Université Lyon I, 69622 Villeurbanne, France \\ 2 L.P.T.M.S, UMR CNRS 8626, Bâtiment 100, Université Paris XI, 91405 Orsay, France
}

(Dated: August 20, 2018)

\begin{abstract}
Hydrodynamic behavior at the vicinity of a confining wall is closely related to the friction properties of the liquid/solid interface. Here we consider, using Molecular Dynamics simulations, the electric contribution to friction for charged surfaces, and the induced modification of the hydrodynamic boundary condition at the confining boundary. The consequences of liquid slippage for electrokinetic phenomena, through the coupling between hydrodynamics and electrostatics within the electric double layer, are explored. Strong amplification of electro-osmotic effects is revealed, and the non-trivial effect of surface charge is discussed. This work allows to reconsider existing experimental data, concerning $\zeta$ potentials of hydrophobic surfaces and suggest the possibility to generate "giant" electro-osmotic and electrophoretic effects, with direct applications in microfluidics.
\end{abstract}

PACS numbers: $68.15+\mathrm{e}, 47.45 . \mathrm{Gx}, 82.45 .-\mathrm{h}$

\section{INTRODUCTION}

With the important development of microfluidic systems, miniaturization of flow devices has become a real challenge [1]. Microchannels are characterized by a large surface-to-volume ratio, so that flows are strongly affected by surface properties. A clear understanding of liquids dynamics close to solid surfaces is consequently an important prerequisite for further progress. Over the recent years, important advances in the rheology of fluids at small scales have been performed, partly thanks to computer simulations, such as Molecular Dynamics (see e.g. 2] and refs. therein), but mainly thanks to the development of new experimental techniques, such as optical velocimetry (see 3, 4] and refs. therein), or dissipation measurements using Surface Force Apparatus and Atomic Force Microscope (see [5, [6] and refs. therein).

In this context, the usual assumption of a no-slip boundary condition for simple liquids at solid surfaces has been critically revisited at small scales in the last years, see e.g. 7] for a review. The conclusions emerging from these studies are that, while the continuum hydrodynamics theory surprisingly remains valid up to very small length scales, the no-slip boundary condition (BC) for the fluid velocity at the solid surface may be violated in many situations (see e.g. [2, [5, 6, 8, [9]). Moreover, it has been shown that this violation of the usual no-slip $\mathrm{BC}$ is controlled by the wetting properties of the fluid on the solid surface: while the no-slip BC is fulfilled on hydrophilic surfaces, a finite velocity slip is measured on hydrophobic surfaces $[2,[5,8]$, originating in a low friction of the liquid at the wall.

In this work, we consider the role of electric properties on liquid-solid friction, a point which has been barely explored up to now 10, 11]. Surfaces indeed usually release charges when in contact with a polar solvent such

*Electronic address: ljoly@lpmcn.univ-lyon1.fr as water, which in turns strongly modifies the liquidsolid interactions at the interface. The natural length scale characterizing the electric interaction range in electrolytes is the so-called Debye length. This length being typically nanometric in standard aqueous electrolytes, one can anticipate that the dynamics of charged systems should probe hydrodynamics in the nanometric vicinity of charged solid surfaces. One can in particular expect an interesting coupling with nanometric slippage, as predicted theoretically for neutral surfaces $[2]$ and evidenced experimentally 5, 8]. Furthermore, hydrophilic and hydrophobic surfaces exhibit different electric properties [12], and the coupling between pure wetting effects and "charge-mediated" effects is a priori subtle and remains to be clarified. Eventually, such an interplay is expected to affect interfacial transport of charges, i.e. electrokinetics, which is commonly used to manipulate liquids in microsystems (e.g. electrophoresis and electro-osmosis). These different points will be considered in the present paper. To study the relevant length scales involved, extensive molecular dynamics (MD) simulations have been used.

In a previous article 13], we presented first results concerning the influence of surface hydrodynamic properties (as encompassed in the so-called hydrodynamic boundary condition) on electrokinetic effects, focusing on streaming current experiments and with a restricted set of electric parameters. Beyond the generalization to other electrokinetic effects, the purpose of the present work is to extend this previous analysis by extensively exploring the influence of the various electric parameters on both the static and the dynamic properties of the surface, therefore rationalizing the interplay between surface charge, hydrodynamics at the interface and electrokinetics response. The paper is organized as follows. In section III we describe our numerical model, together with some details of the simulation procedure. We focus in section [II on the static properties of our systems. We then turn to the dynamic behaviour and explore in section [V] the coupling between liquid/solid friction and surface charge. The 


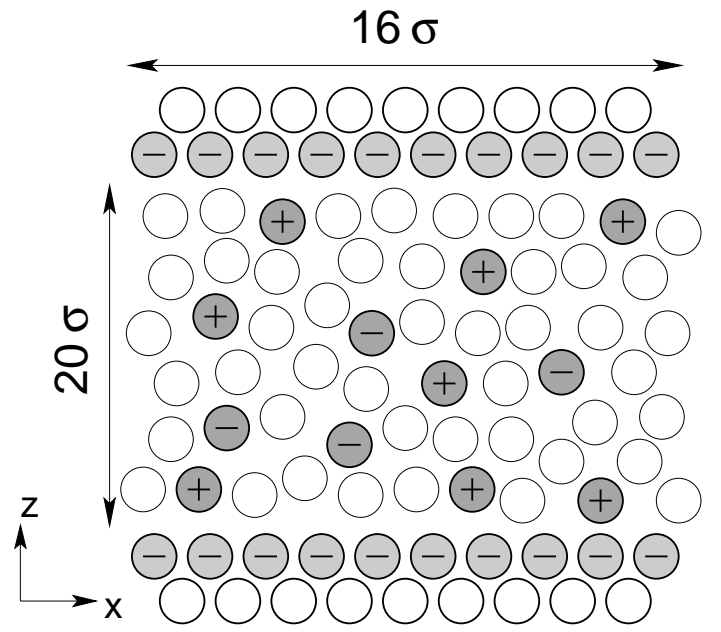

FIG. 1: Simulated system. The simulation cell extends over $16 \sigma$ along the $\mathrm{O} y$ direction, perpendicular to the figure.

reciprocal coupling between hydrodynamics and electric properties of the interface is studied in section $\nabla$ A strong amplification of electrokinetic effects in the presence of slip at the solid surface is reported. Finally experimental consequences of this work are addressed in section VI with focus on the origin of $\zeta$ potential on hydrophobic surfaces, and the possibility to strongly amplify electrokinetics effects using polarized hydrophobic surfaces, with direct applications in microfluidics.

\section{MODEL AND PARAMETERS}

We first describe our microscopic model (see Fig. 1) and some details of the simulation procedure. The fluid system (solvent and microions) is confined between two parallel solid substrates, themselves composed of individual atoms fixed on an fcc lattice. The solvent and solid substrate particles interact via Lennard-Jones (LJ) potentials,

$$
v_{i j}(r)=4 \epsilon\left[\left(\frac{\sigma}{r}\right)^{12}-c_{i j}\left(\frac{\sigma}{r}\right)^{6}\right]
$$

with identical interaction energies $\epsilon$ and molecular diameters $\sigma$. The tunable parameters $c_{i j}$ enable us to adjust the wetting properties of the fluid on the substrate 2]: for a given fluid-fluid cohesion $c_{F F}$, the substrate displays a "hydrophilic" behavior for large fluid-solid cohesivity, $c_{F S}$, and a "hydrophobic" behavior for small $c_{F S}$. Here, the wetting (respectively non-wetting) situation is typically achieved by taking $c_{F S}=1$ (resp. 0.5) for a fixed $c_{F F}=1.2$. This leads to a contact angle $\theta$ of a liquid droplet on the substrate, measured in the simulations, equal to $80^{\circ}$ (resp. $140^{\circ}$ ) for a temperature $k_{\mathrm{B}} T / \epsilon=1$ (see 2] for an exhaustive discussion on this point).

Besides, microions interact through both LennardJones potentials as described in Eq. (1) and Coulomb potential in a medium with dielectric permittivity $\varepsilon_{\mathrm{d}}$ :

$$
v_{\alpha \beta}(r)=k_{\mathrm{B}} T q_{\alpha} q_{\beta} \frac{\ell_{\mathrm{B}}}{r},
$$

where $q_{\alpha}$ and $q_{\beta}$ are the valences of the interacting charges, and $\ell_{\mathrm{B}}=e^{2} /\left(4 \pi \varepsilon_{\mathrm{d}} k_{\mathrm{B}} T\right)$ is the Bjerrum length, $e$ denoting the elementary charge (the Bjerrum length is the typical scale at which thermal energy and electric interaction energy compare; in water at room temperature $\left.\ell_{\mathrm{B}} \approx 0.7 \mathrm{~nm}\right)$. The influence of the solvent permittivity on static and dynamical properties will be tested by varying $\ell_{\mathrm{B}}$ in the parameter range $[0.25 \sigma ; 5 \sigma]$; otherwise we will choose $\ell_{\mathrm{B}}=\sigma$ as the default value. Note that we consider a case where LJ parameters are identical for charged and neutral atoms (liquid or solid); as we verified in appendix [B] this simplifying assumption enables us to clearly separate hydrodynamic and electrostatic properties, without affecting the generic mechanisms evidenced in this work.

Wall atoms are organized into five layers of an fcc solid (in the 100 direction) in both walls. For each wall, only the first layer, which is in contact with the fluid, is charged. The corresponding $N_{\text {wall }}$ atoms bear a discrete charge, with valency $q_{\text {wall }}=-Z / N_{\text {wall }}$ so that each wall bears a negative net charge $-Z e$. The solvent contains $2 Z$ monovalent counterions, to which $N_{\mathrm{s}}=N_{+}+N_{-}$salt ions are added, all with unit valence. Global electroneutrality is enforced by imposing $N_{+}=N_{-}$. The simulated systems are generally made up of $10^{4}$ atoms. A typical solvent density is $\rho_{\mathrm{f}} \sigma^{3} \sim 0.9$, while the concentration of microions $\rho_{\mathrm{s}}=N_{ \pm} / \mathcal{V}$ will be varied between $\rho_{\mathrm{s}} \sigma^{3}=5 \times 10^{-3}$ and $\rho_{\mathrm{s}} \sigma^{3}=0.16$ (with $\mathcal{V}$ the total volume of the sample). With a typical value $\sigma=0.5 \mathrm{~nm}$, this corresponds roughly to an ionic strength varying between $10^{-2} \mathrm{M}$ and $1 \mathrm{M}$. For $\ell_{\mathrm{B}}=\sigma$, the corresponding Debye screening length (see below) ranges from a few $\ell_{\mathrm{B}}$ to a fraction of $\ell_{\mathrm{B}}$. Saltfree situations have also been investigated and will be reported below. The influence of surface charge will be considered, by varying the charge per unit surface $-\Sigma$ in the parameter range $\left[-0.02 e / \sigma^{2} ;-0.8 e / \sigma^{2}\right]$ (note that for convenience we define the parameter $\Sigma$ to be the negative of the surface charge). Unless otherwise stated, we will choose $\Sigma=0.2 e / \sigma^{2}$, with a corresponding Gouy-Chapman length $\ell_{\mathrm{GC}}=1 /\left(2 \pi \ell_{\mathrm{B}}|\Sigma|\right)=0.8 \sigma$. For $\sigma=0.5 \mathrm{~nm}$, this translates into a typical surface density $-0.13 \mathrm{C} / \mathrm{m}^{2}$. For $\ell_{\mathrm{B}}=\sigma$ and the salt concentrations considered here, the surface potential $V_{0}$ ranges between $e V_{0} \approx k_{\mathrm{B}} T$ and $e V_{0} \approx 4 k_{\mathrm{B}} T$, allowing us to explore both linear (Debye-Hückel-like) and non-linear situations. Periodic boundary conditions are applied in the $x$ and $y$ directions with $L_{x}=L_{y}=16 \sigma$, and the distance between the walls is $L_{z}=20.9 \sigma$. Ewald sums are used to compute Coulombic interactions (assuming a periodicity in the $z$ direction with a box size of $5 L_{z}$ ) [44]. In the subsequent analysis, Lennard-Jones units are used, with a characteristic distance $\sigma$ and time $\tau=\left(m \sigma^{2} / \epsilon\right)^{1 / 2}$. Temperature is kept constant to $k_{\mathrm{B}} T=1$ by applying a 
Hoover drag to the $y$ degrees of freedom only, i.e. in the direction perpendicular to the flow and confinement [2].

Our model therefore includes the discrete nature of the solvent and charges and a tuning wettability of the surface, whereas these effects are usually neglected in the traditional description of electrokinetic phenomena. We chose to describe Coulombic interactions at the level of an effective dielectric medium (with dielectric permittivity $\varepsilon_{\mathrm{d}}$ ). This simplifying assumption - which could be relaxed using a more realistic model for the solvent [14] enables us to investigate specifically the generic interplay between hydrodynamic and electrostatic effects, which is the main focus of this work. We expect the conclusions obtained to be generically valid.

In order to model the contribution of electric charges to friction, we will explore the influence of various electric parameters: electrolyte concentration $\rho_{\mathrm{s}}$, surface charge $\Sigma$ and permittivity of the solvent, via the Bjerrum length $\ell_{\mathrm{B}}$. To be specific, we performed different sets of simulations, including:

- varying salt concentration $\left(\rho_{\mathrm{s}} \sigma^{3} \in\left[5 \times 10^{-3} ; 0.16\right]\right)$, with fixed Bjerrum length $\left(\ell_{\mathrm{B}}=\sigma\right)$ and surface charge $\left(\Sigma=0.2 e / \sigma^{2}\right)$;

- varying surface charge $\left(\Sigma \sigma^{2} / e \in[0.02 ; 0.8]\right)$, with fixed Bjerrum length $\left(\ell_{\mathrm{B}}=\sigma\right)$ and salt concentration $\left(\rho_{\mathrm{s}} \sigma^{3}=0.06\right)$;

- varying Bjerrum length $\left(\ell_{\mathrm{B}} / \sigma \in[0.25 ; 5]\right)$, with fixed surface charge $\left(\Sigma=0.2 e / \sigma^{2}\right)$ and no electrolyte. In addition, we finally considered the effect of doubling the Bjerrum length $\left(\ell_{\mathrm{B}}=2 \sigma\right)$ in the presence of salt, with $\rho_{\mathrm{s}} \sigma^{3}=0.06$ and $\Sigma=0.2 e / \sigma^{2}$.

\section{STATIC}

We now turn to the results of the simulations. We first focus on the equilibrium properties of the charged interfaces. As a rule, a solid surface immersed in an electrolyte solution develops spontaneously an electric charge, under the action of several mechanisms: dissociation of ionisable groups, release of ionic impurities, specific adsorption of charged species present in the solution, etc. In response to this surface charge, the microions of the liquid rearrange themselves to form, in the vicinity of the solid surface, a diffuse layer named Electric Double Layer (EDL), carrying a net charge opposite to that of the surface. The so-called Debye length, denoted here $\kappa^{-1}$, provides a measure of the EDL extension and determines the electric interaction range between macromolecules. This characteristic length plays a crucial role in the static phase behavior of these systems [15].

The standard description of the microions cloud in the vicinity of a charged surface, in the framework of the Gouy-Chapman theory, involves a Poisson description of the electrostatics $\left(\Delta V+\rho_{\mathrm{e}} / \varepsilon_{\mathrm{d}}=0\right.$, with $V$ the electric potential and $\rho_{\mathrm{e}}=e\left(\rho_{+}-\rho_{-}\right)$the charge density, defined

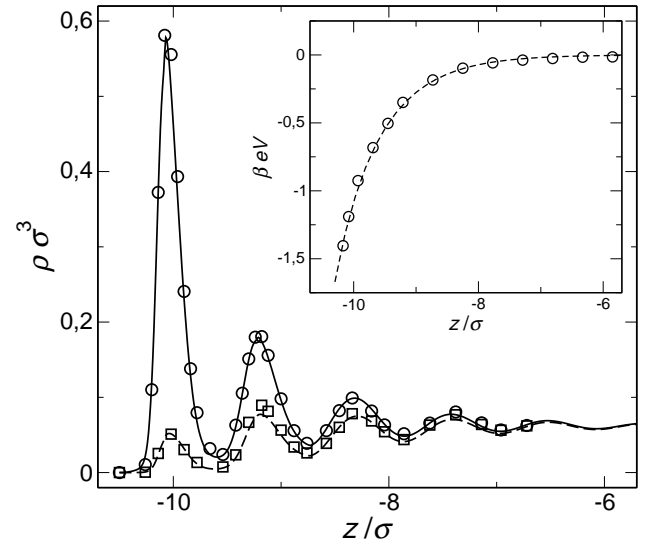

FIG. 2: Microionic density profiles, averaged over the $x y$ directions $\left(\rho_{\mathrm{s}} \sigma^{3}=0.06, \Sigma=0.2 e / \sigma^{2}, \ell_{\mathrm{B}}=\sigma\right.$, wetting case). Symbols: Molecular Dynamics results for the counter-ions (o) and co-ions $(\square)$; solid and dashed lines correspond to the predictions of the modified PB description (see text). The inset shows the electrostatic potential. Symbols (o): Molecular Dynamics results calculated from Poisson's equation and the measured microions profiles; dashed line: bare PB prediction (see text). The position of the wall, defined as that of the centers of the last layer of wall atoms, is located at $z_{\mathrm{wall}}=-10.9 \sigma$.

in terms of the microions concentrations), coupled with a Boltzmann equilibrium description for the microions distribution $\left(\rho_{ \pm}=\rho_{\mathrm{s}}^{\text {bulk }} \exp (\mp \beta e V)\right.$, with $\beta=1 / k_{\mathrm{B}} T$ and $\rho_{\mathrm{s}}^{\text {bulk }}$ the bulk microions concentration), leading to the standard Poisson-Boltzmann (PB) equation for the electric potential $V$ in the EDL [16]:

$$
\beta e \Delta V=\kappa^{2} \sinh (\beta e V)
$$

where $\kappa^{-1}=\left(8 \pi \ell_{\mathrm{B}} \rho_{\mathrm{s}}^{\text {bulk }}\right)^{-1 / 2}$ is the Debye screening length. In situations of extreme confinement where the notion of bulk becomes irrelevant, $\rho_{\mathrm{s}}^{\text {bulk }}$ should be viewed as a normalization density, or equivalently as the salt density in a salt reservoir against which the solution is dialyzed.

In Fig. 2] we show typical density profiles of the microions close to one of the confining surfaces. Important structuration effects can be observed close to the charged surface. This is a priori incompatible with the PoissonBoltzmann (PB) prediction [16]: within PB, an oscillating density profile implies that the electric field vanishes at every extrema, which corresponds to a change of sign of the integrated charge. This in turns implies that the system exhibits overcharging. Such an effect can be rigorously ruled out within mean-field descriptions, such as the $\mathrm{PB}$ one [17]. However, the oscillations in the microions profiles exhibited here are not associated with any charge inversion, but result from the structuration of the solvent itself. Such an effect can be captured by a modified PB description. Indeed, due to the presence of the solvent particles, the microions not only organize themselves due to electrostatic interactions (which corre- 
sponds to the usual PB description) but also due to the effective external field associated with the structuration in the solvent, $V_{\text {ext }}(z)=-k_{\mathrm{B}} T \log \left[\rho_{\mathrm{f}}(z) / \rho_{\mathrm{f}}\right]$, with $\rho_{\mathrm{f}}(z)$ the solvent density profile and $\rho_{\mathrm{f}}$ its bulk value. The microions density profiles $\rho_{ \pm}(z)$ correspondingly obey a modified Boltzmann equilibrium:

$$
\rho_{ \pm}(z) \propto e^{\beta\left(\mp e V(z)-V_{\text {ext }}(z)\right)} \propto \rho_{\mathrm{f}}(z) e^{\mp \beta e V(z)} .
$$

Such a relationship emerges naturally from a simple Density Functional Theory, that allows to rationalize the argument, accounting for the the discrete nature of both solvent and charged atoms exactly, while the standard mean-field PB free energy is assumed for the electrostatic part. The details can be found in appendix [

Inserting Eq. (4) into Poisson equation, we find that the electrostatic potential follows a modified PB equation,

$$
\beta e \Delta V=\kappa^{2} \gamma(z) \sinh (\beta e V)
$$

where $\gamma(z)=\rho_{\mathrm{f}}(z) / \rho_{\mathrm{f}}$ is the normalized solvent density profile. This equation allows for instance to compute the electric potential once the solvent density is known, but should be supplemented with a closure relation to predict both $\rho_{f}(z)$ and $V(z)$. A more modest goal is to test the relevance of this approach by measuring the fluid density profiles, $\rho_{\mathrm{f}}(z)$, and subsequently solving Poisson equation with the microionic densities given by Eq. (4). As shown in Fig. 2] this procedure leads to results that are in remarkable agreement with simulations profiles obtained from Molecular Dynamics. Moreover, a further useful approximation can be proposed : the solution of the modified $\mathrm{PB}$ equation for the electrostatic potential is as a matter of fact very close by the "bare" PB solution $V_{\mathrm{PB}}(z)$ (corresponding to $\gamma(z)=1$ ), whose analytic expression can be found in the literature [15, 16, 18]. This leads to $\rho_{ \pm}(z) \propto \rho_{\mathrm{f}}(z) \exp \left[\mp \beta e V_{\mathrm{PB}}(z)\right]$. The validity of this approximation - surprising in view of the strong layering effect at work - is emphasized in Fig. 2 (inset), where the corresponding bare $\mathrm{PB}$ potential [18] is plotted against the "exact" electrostatic potential. The latter is obtained from the simulations using Poisson's equation by integrating twice the charge density profile $\rho_{\mathrm{e}}=e\left(\rho_{+}-\rho_{-}\right)$.

The case of no-added salt gives another interesting limiting case, which we now consider. In the no-salt case, only counterions are present in the solution, with a total charge compensating exactly the charge of both surfaces. The PB equation becomes:

$$
\beta e \Delta V=-4 \pi \ell_{\mathrm{B}} e \rho_{0} \mathrm{e}^{-\beta e V},
$$

where $\rho_{0}$ represents a reference concentration for counterions, for which $V=0$. This equation can be solved analytically for the electric potential $V_{\mathrm{PB}}$ and the counterions concentration $\rho_{\mathrm{i}}$ in the simple case of an electrolyte confined between two parallel plates, as considered in our simulations 16]. As in the presence of salt, we can extend the $\mathrm{PB}$ prediction to take into account the effective

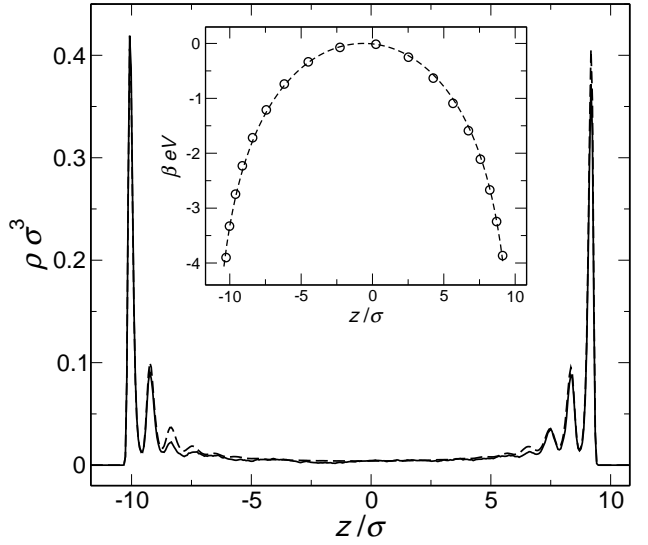

FIG. 3: Counterions density profile, averaged over the $x y$ directions, without added electrolyte $\left(\Sigma=0.2 e / \sigma^{2}, \ell_{\mathrm{B}}=\sigma\right.$, wetting case). (-): MD results; (--): prediction of the modified PB description, using the bare PB potential (see text). Inset: electrostatic potential. Symbols (o): MD results calculated from Poisson's equation and counterions profiles; dashed line: bare PB prediction (see text). The walls are located at $z_{\text {wall }}^{\text {inf }}=-10.9 \sigma$ and $z_{\text {wall }}^{\text {sup }}=10.0 \sigma$.

external field due to the structuration of the solvent, using the very same approach. The results for a typical no-salt configuration are presented on Fig. 3. We can check that the bare PB prediction perfectly accounts for the electric potential, for which no structuration can be seen. The counterions density profile is accordingly well described by $\rho_{\mathrm{i}}(z)=\rho_{\mathrm{f}}(z) \exp \left[-\beta e V_{\mathrm{PB}}(z)\right]$.

Globally we have tested the validity of this modified $\mathrm{PB}$ approach in our simulations with various conditions, involving a broad range of the different parameters, salt concentration $\rho_{\mathrm{s}}$, surface charge $\Sigma$ and Bjerrum length $\ell_{\mathrm{B}}$. The modified PB approach has been found to describe with accuracy every situation considered, over the whole parameter range for both surface charge and salt concentration (not shown). However, we found that the modified PB prediction breaks down at large Bjerrum length (we considered two cases with $\ell_{\mathrm{B}}=2.24 \sigma$ and $\ell_{\mathrm{B}}=5.04 \sigma$, not shown). This failure is however expected : for large Bjerrum length, microionic correlations become important and invalidate the modified PoissonBoltzmann ansatz. A simple analysis, discussed in details at the end of appendix $\mathrm{C}$ shows that the criterion for this failure can be written $(\Sigma / e) \ell_{\mathrm{B}}^{2}>1$.

\section{DYNAMICS}

\section{A. Wetting versus non-wetting : the Poiseuille test-bench}

We now come to the dynamical aspects. As a first step, we briefly recall how the hydrodynamics at the interface, as characterized by the hydrodynamic boundary condi- 


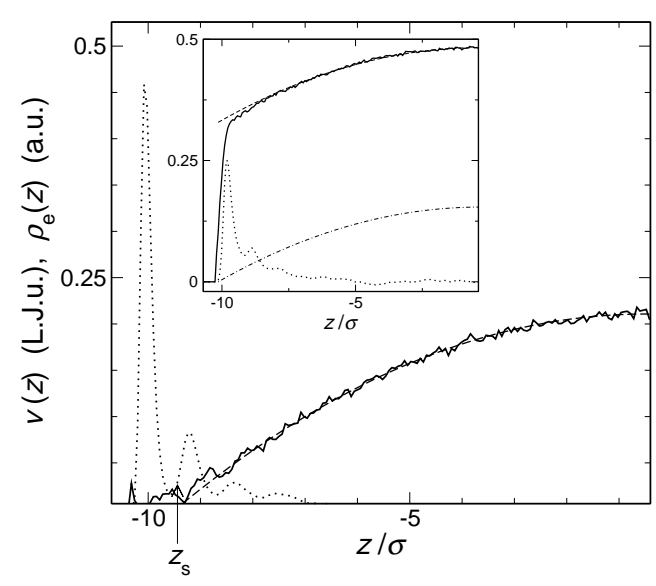

FIG. 4: Measured Poiseuille velocity profile (solid line) in the wetting case $\left(c_{F S}=1, \ell_{\mathrm{B}}=\sigma, \Sigma=0.2 e / \sigma^{2}, \rho_{\mathrm{s}} \sigma^{3}=0.06\right)$. Dashed line: hydrodynamic prediction using a no-slip BC at the 'plane of shear' located at $z_{\mathrm{s}}$ (indicated by the arrow). To emphasize the existence of an immobile Stern layer, we also indicate the charge density profile $\rho_{\mathrm{e}}(z)=e\left(\rho_{+}(z)-\rho_{-}(z)\right)$ (dotted line), with arbitrary units. The position of the wall (defined as that of the centers of the last layer of wall atoms) is at $z_{\text {wall }}=-10.9 \sigma$. Inset: Results for the non-wetting case $\left(c_{F S}=0.5\right)$. Solid line: velocity profile measured in the simulation (shown on the same scale as in the main graph); dashed line: hydrodynamic prediction with a partial slip BC, with a slip length $b \approx 11 \sigma$; dashed-dot line: hydrodynamic prediction with a no-slip BC; dotted line: charge density profile (arbitrary units).

tion (HBC), is affected by a modification of the wetting properties of the surfaces (see [2, 19, 20] for a more detailed discussion); We then turn to the specific role of electric parameters.

We chose to probe the HBC in a Poiseuille configuration, by applying an external force per particle $f_{0}$, in the $x$ direction, to all microscopic particles. The Bjerrum length and surface charge are set to their default values $\left(\ell_{\mathrm{B}}=\sigma\right.$ and $\left.\Sigma=0.2 e / \sigma^{2}\right)$, and the salt concentration is varied between $\rho_{\mathrm{s}} \sigma^{3}=5 \times 10^{-3}$ and $\rho_{\mathrm{s}} \sigma^{3}=0.16$. We start by discussing the measured velocity profiles. The situation corresponding to a wetting substrate - with $c_{F S}=1$ in Eq. (11) - is shown in the main plot of Fig. 4 (here for $f_{0}=0.02$ in LJ units). The velocity profile shows a parabolic shape as predicted by continuum hydrodynamics, even at the EDL level. Moreover, the viscosity, deduced from the curvature of the parabolic shape, retains its bulk value. Nevertheless, our measurements have shown that the no-slip BC applies inside the liquid, at a distance of about one layer of solvent particle. This observation is consistent with previous theoretical and experimental predictions [2, 20]. This position of the no-slip BC here defines the "plane of shear" position, $z_{\mathrm{s}}$, usually introduced in the electrokinetic literature [18]. We note that $z_{\mathrm{s}}$ does not vary significantly with the salt concentration, in the parameter range investigated.

On the other hand, the non-wetting case displays a very different behavior, as shown in the inset of Fig. [4 A non-wetting substrate is set up by choosing a smaller value of $c_{F S}$, here $c_{F S}=0.5$. First, concerning the velocity profile, a large amount of slip is found at the wall surface, in accordance with observations on non-wetting surfaces [2]. More quantitatively, slippage is characterized by a slip length, $b$, defined as the distance at which the linear extrapolation of the velocity profile vanishes. In other words, this amounts to replace the no-slip BC by a partial slip $\mathrm{BC}$, defined as $b \partial_{z} v=v$ at the wall position [20]. As shown in the inset of Fig. [4 the velocity profile is well fitted by the continuum hydrodynamics (parabolic) prediction, together with a partial slip $\mathrm{BC}$, characterized by a non vanishing slip length (here $b \approx 11 \sigma$ ). The measured slip length $b$ barely depends on the salt concentration, a point which we now rationalize.

\section{B. Rationalizing friction and slipping}

Slippage can be interpreted in terms of friction properties at the liquid/solid interface. Indeed, the usual partial slip BC can be interpreted as the continuity of tangential stress at the liquid/solid interface: the viscous shear stress exerted by the liquid on the wall $\eta \partial_{z} v$ (where $\eta$ is the liquid viscosity) is equal to the friction stress suffered by the liquid from the wall, which can be written in the form $\sigma_{x z}=\lambda v_{\mathrm{s}}$, where $\lambda$ is the interfacial friction coefficient, linking the friction stress $\sigma_{x z}$ and the relative liquid/solid slip velocity $v_{\mathrm{s}}$. This equality corresponds to the partial slip $\mathrm{BC} v_{\mathrm{s}}=b \partial_{z} v$, with the slip length $b=\eta / \lambda$. This expression formalizes the simple idea that liquids slip all the more as the interfacial friction is low. It also means that the slip length characteristics can be directly deduced from a friction analysis at the liquid/solid interface.

The dependence of the slip length on the electric parameters can then be rationalized on the basis of a simple argument based on the influence of the electric interaction on the friction coefficient. We start from a Green-Kubo expression for the interfacial friction coefficient [20]:

$$
\lambda=\frac{\eta}{b}=\frac{1}{\mathcal{A} k_{\mathrm{B}} T} \int_{0}^{\infty}\left\langle F_{x}(t) F_{x}(0)\right\rangle \mathrm{d} t,
$$

where $\mathcal{A}=L_{x} L_{y}$ is the area of the solid surface under consideration, and $F_{x}$ is the $O x$ component of the instantaneous force exerted by the wall on the liquid at equilibrium. Apart from a few simple situations, it is difficult to evaluate this expression. In this study we therefore restrict ourselves to extracting scaling laws for the slip length $b$. We start with the evaluation of an order of magnitude for the temporal auto-correlation integral:

$$
\int_{0}^{\infty}\left\langle F_{x}(t) F_{x}(0)\right\rangle \mathrm{d} t=F_{x}^{2} \tau_{D}
$$

where $F_{x}=\sqrt{\left\langle F_{x}^{2}(t)\right\rangle}$ is the r.m.s. force, and $\tau_{D}$ the relaxation time scale of the force correlation function. 
The latter can be estimated as the diffusion time of the liquid molecules over the characteristic wavelength of the wall corrugation $\ell_{\mathrm{r}}$ 19]:

$$
\tau_{D}=\frac{\ell_{\mathrm{r}}^{2}}{D}
$$

where $D$ is the self-diffusion coefficient of liquid molecules. The validity of this estimate has been exhaustively tested in Ref. [19.

We now separate Lennard-Jones and electric contributions to the total force: $F_{x}=F_{\mathrm{LJ}}+F_{\mathrm{ES}}$. Using Eq. (7), we then write: $1 / b \propto\left(F_{\mathrm{LJ}}+F_{\mathrm{ES}}\right)^{2} \propto F_{\mathrm{LJ}}^{2}+F_{\mathrm{LJ}} F_{\mathrm{ES}}+F_{\mathrm{ES}}^{2}$. In every performed simulations, we noticed that the slip properties were only slightly affected by the presence of charge; we can therefore assume that the electric contribution to friction is small compared to the Lennard-Jones term; it is then possible to neglect the pure electric contribution (the $F_{\mathrm{ES}}^{2}$ term), which leads to:

$$
\frac{1}{b}=\frac{1}{b_{\mathrm{LJ}}}+\frac{1}{b^{\prime}}
$$

with $1 / b^{\prime} \propto F_{\mathrm{LJ}} F_{\mathrm{ES}}$. We can then estimate an order of magnitude for the first order electric contribution to friction: $1 / b^{\prime} \propto F_{\mathrm{ES}} \sim Q \times E$, where $Q=\Sigma \mathcal{A}$ is the total charge of the EDL (compensating the surface charge), and $E=\Sigma / \varepsilon_{\mathrm{d}} \sim \Sigma \ell_{\mathrm{B}}$ the electric field at the interface. We finally find that the $b^{\prime}$ contribution to slippage varies as:

$$
b^{\prime} \sim \Sigma^{-2} \ell_{\mathrm{B}}^{-1},
$$

which we rewrite $b^{\prime} / \sigma=\alpha\left(\Sigma \sigma^{2} / e\right)^{-2}\left(\ell_{\mathrm{B}} / \sigma\right)^{-1}, \alpha \sim 1$ a numerical prefactor. The electric contribution to the slip length therefore does not depend on the Debye length of the system. Alltogether, we obtain the following prediction for the slip length $b$ on the surface charge :

$$
b=\frac{b_{L J}}{1+\frac{\Sigma^{2}}{e^{2}} \ell_{\mathrm{B}} \sigma^{2} b_{L J}}
$$

Note that the dependence of the Lennard-Jones contribution to the slip length, $b_{L J}$, on the microscopic parameters can also be predicted, see Ref. [19].

\section{Comparison of simulation data against theory for slip length and shear plane position}

We have tested the above description against MD results. To this end, we extracted the first order electric contribution $b^{\prime}$ from the simulation results, using Eq. (10); to this purpose, the LJ contribution was measured in complementary simulations, using identical systems without charges (with identical pressure). The inset of Fig. 5 presents the numerical results for $b^{\prime}$ as a function of $\Sigma^{2} \ell_{\mathrm{B}}$, using various $\Sigma$ and $\ell_{\mathrm{B}}$, which are perfectly fitted with the prediction of our simple model: $b^{\prime} / \sigma=1.10\left(\Sigma \sigma^{2} / e\right)^{-2}\left(\ell_{\mathrm{B}} / \sigma\right)^{-1}$. Only with the highest

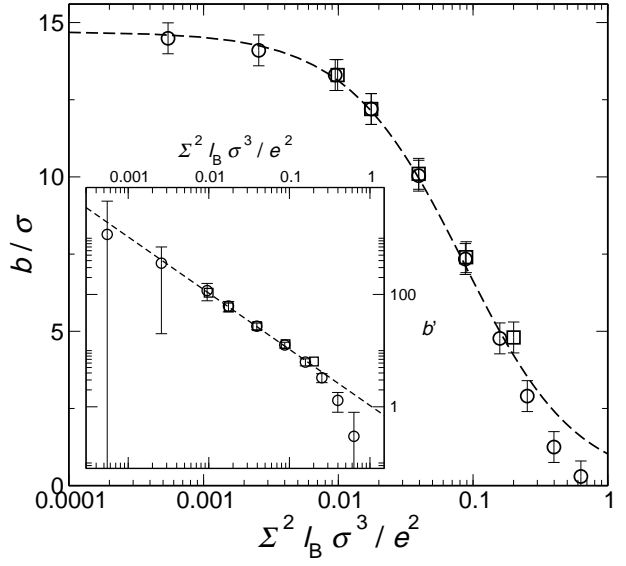

FIG. 5: Slip length $b$ as a function of $\Sigma^{2} \ell_{\mathrm{B}}$, in the non-wetting case. Symbols: MD results with fixed Bjerrum length $\ell_{\mathrm{B}}=\sigma$ and varying surface charge $(\circ)\left(\rho_{\mathrm{s}} \sigma^{3}=0.06\right)$, or fixed surface charge $\Sigma=0.2 e / \sigma^{2}$ and varying Bjerrum length, without added salt ( $\square)$. Dashed line: analytical fit using Eq. (12) (see text). Inset: First order electric contribution $b^{\prime}$ as a function of $\Sigma^{2} \ell_{\mathrm{B}}$. Symbols: MD results (symbols are identical to those of the main graph). Uncertainties on results with varying Bjerrum length, not represented for the sake of clarity, are comparable to the size of the symbols. The dashed line has a slope -1 . The agreement is excellent except for very high surface charge (see text).

surface charges does a slight discrepancy appear [45]. We were thus able to account precisely for the complete slip length $b$ with an analytical expression, Eq. (12), simply by adding the LJ and electric contributions, as can be seen on the main graph of Fig. 5. Finally we have checked that the slip length is independent on the salt concentration at fixed $\Sigma$ and $\ell_{\mathrm{B}}$ (not shown), again in accordance with our simple model.

In the wetting case, no slip occurs at the surfaces; yet we can consider the position of the no-slip plane $z_{\mathrm{s}}$, defining equivalently the width of the immobile Stern layer (see section V). Figure 6 shows the evolution of $z_{\mathrm{s}}$ with $\Sigma$ (main graph) and $\ell_{\mathrm{B}}$ (inset). Using a Green-Kubo expression similar to the one used for $b$ [20] and following the same derivation as above, one expects $z_{\mathrm{s}} \sim \Sigma^{2} / \ell_{\mathrm{B}}$. In spite of the rather important measurement noise, this prediction is rather well verified as can be seen from figure [6] where $z_{\mathrm{s}}(\Sigma)$ is fitted by $z_{\mathrm{s}} / \sigma=0.94+2.27\left(\Sigma \sigma^{2} / e\right)^{2}$ and $z_{\mathrm{s}}\left(\ell_{\mathrm{B}}\right)$ by $z_{\mathrm{s}} / \sigma=1.01+0.145 \ell_{\mathrm{B}} / \sigma$. Finally, we recall that no effect of salt concentration on $z_{\mathrm{s}}$ has been observed.

\section{ELECTROKINETICS}

In section IV we have shown that the traditional noslip BC for the liquid velocity at the wall was violated on non-wetting surfaces, with a small but finite slip length ( $b \sim 10 \sigma$, corresponding to $b \sim 5 \mathrm{~nm}$ ), even for highly charged surfaces (significant slip until $\Sigma \sim-0.3 e / \sigma^{2}$, 


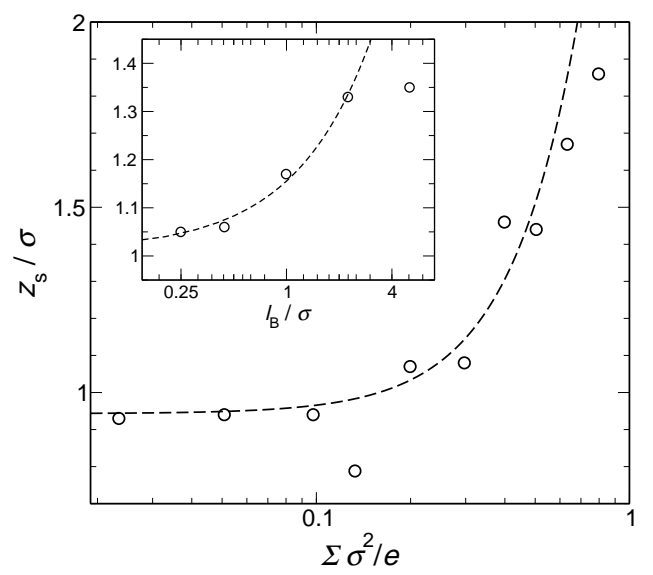

FIG. 6: Width of the immobile Stern layer $z_{\mathrm{s}}$ as a function of surface charge $\Sigma$ (wetting case, $\ell_{\mathrm{B}}=\sigma, \rho_{\mathrm{s}} \sigma^{3}=0.06$ ). Symbols: MD results; dashed line: analytical fit with a simple quadratic expression (see text). Inset: $z_{\mathrm{s}}$ as a function of Bjerrum length $\ell_{\mathrm{B}}$ (wetting case, no salt, $\Sigma=0.2 e / \sigma^{2}$ ). Symbols: MD results; dashed line: simple analytical fit.

corresponding to $\Sigma \sim 0.2 \mathrm{C} / \mathrm{m}^{2}$ and $\left.V_{0} \sim 80 \mathrm{mV}\right)$. We now investigate the consequences of these modifications of hydrodynamics at the interfaces for the dynamics of charged systems, and particularly for electrokinetic phenomena, commonly used to manipulate liquids in microsystems (e.g. electrophoresis and electro-osmosis). After a brief recall of electrokinetic effects, we will perform streaming current and electro-osmosis simulations, in order to explore the influence of such hydrodynamic slippage. Finally, we will focus on the specific role of electric parameters.

\section{A. Zeta potential}

In addition to its interest for the understanding of static properties of charged systems, the EDL is, on the dynamical level, at the origin of numerous electrokinetic phenomena: electrophoresis, electro-osmosis, streaming current or potential, etc. Because these various effects take their origin at the surface of the sample via the EDL, they provide smart and particularly efficient ways to drive or manipulate flows in microfluidic devices 1, 21], where surface effects become predominant.

The extension of the EDL is typically on the order of a few nanometers and electrokinetic phenomena therefore probe the nanorheology of the solvent+ions system at the charged surface. This can thus raise some doubts regarding the validity of continuum approaches to describe the dynamics at such scales. Those doubts seem particularly relevant concerning the traditional description of the EDL dynamics, which relies both on the mean-field Poisson-Boltzmann theory of the microion clouds, but also on continuum hydrodynamics for the flow fields [18]. These two aspects are embodied in the so-called zeta po- tential, denoted $\zeta$, which is traditionally defined as the electric potential $V\left(z_{\mathrm{s}}\right)$ computed at the surface of shear $z_{\mathrm{s}}$, where the fluid velocity vanishes. This quantity plays a key role in electrokinetic phenomena [14, 18, 22, 23], since it quantifies the coupling between flow characteristics in the solvent (via the mean velocity or applied pressure drop) and electric quantities (electric field, induced streaming current or potential). An important point is that the standard electrokinetic description is based on the assumption of a no-slip boundary condition of the liquid at the solid interface.

In section III we showed that the traditional PB description is relevant for the electric potential, even at the EDL scale. Yet in section [IV] we observed that although a continuum hydrodynamic approach stands down to EDL sizes, the no-slip BC could be violated in non-wetting situations, in accordance with previous numerical [2] and experimental [5, 8] work. We expect this modification of the interfacial hydrodynamics to affect electrokinetic properties of charged interfaces, as it was demonstrated in a previous work [13], focused on streaming current simulations, and where only a limited set of electric parameters have been investigated. We will now extend this work to other electrokinetic phenomena, exploring extensively the influence of various electric parameters.

\section{B. Streaming current}

First of all, we note that the Poiseuille configurations implemented in the preceding section to probe the $\mathrm{HBC}$ corresponds directly to streaming current experiments: the external force $f_{0}$ accounts for the pressure gradient, and we can measure the electric current, $I_{\mathrm{e}}$, associated with the convective motion of the microions.

The standard EDL description of this electrokinetic effect predicts a linear relationship between the current and the force, in the form [18]:

$$
I_{\mathrm{e}}=-\frac{\varepsilon_{\mathrm{d}} \zeta}{\eta} \mathcal{A} f_{0},
$$

where $\eta$ is the shear viscosity of the fluid and $\mathcal{A}$ the fluid slab cross area. Linear response (in the applied force) was carefully checked in our simulations. In the following we use this expression as the definition of the $\zeta$ potential, in line with experimental procedures.

In the wetting case, we have seen that the liquid velocity vanishes inside the liquid, at a distance $z_{\mathrm{s}}$ of about one layer of solvent particles (see section [V). As shown in Fig. 4 where the charge density profile, $\rho_{\mathrm{e}}(z)$, is plotted against distance, the first layer of microions, located within $z_{\mathrm{s}}$, does not contribute to the convective transport, thereby reducing the global streaming current. This first layer coincides with the so-called Stern layer of immobile microions close to the charged surface [18].

In the non-wetting case, the liquid was shown to slip significantly at the wall, with a complete disappearance 


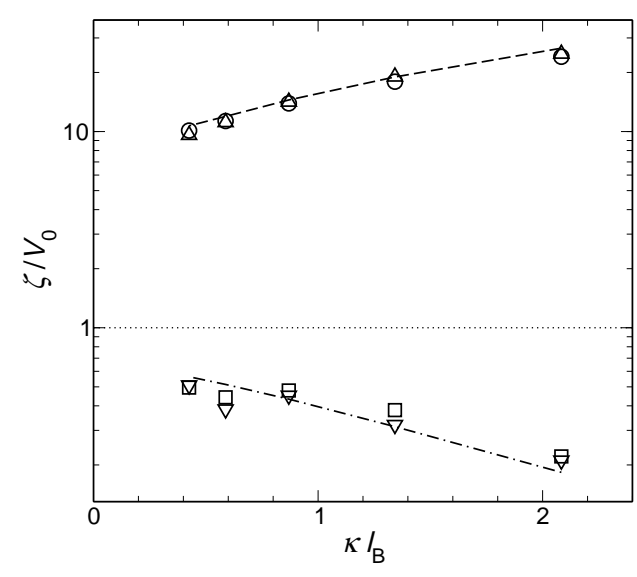

FIG. 7: Measured $\zeta$ potential as a function of the screening factor $\kappa \ell_{\mathrm{B}}$ in streaming current ( $\square$ : wetting case; $\circ$ : nonwetting case) and electro-osmosis $(\nabla$ : wetting case; $\triangle$ : nonwetting case) simulations. The $\zeta$ potential is normalized by the bare surface potential $V_{0}$ obtained from the PB expression at a given $\kappa$ and surface charge (see the discussion on the inset of Fig. 2). For the wetting case (bottom), the dashed-dotted line is the PB electrostatic potential $V\left(z_{\mathrm{s}}\right)$, where the 'plane of shear' position $z_{\mathrm{s}}$ does not vary significantly with salt. For the non-wetting case (top), the dashed line corresponds to the slip prediction Eq. (15), with $b=11 \sigma$.

of the immobile Stern layer (see section [IV). Concerning microions transport, an important point here is that the first layer of microions now contributes by a large amount to the global streaming current, at variance with the wetting case. In other words, the plane of shear position $z_{\mathrm{s}}$ is now virtually located beyond the wall, and the Stern layer has completely disappeared. The remobilization of the Stern layer adds on to the slippage effect and contributes significantly to the increase of the electric current measured for hydrophobic surfaces.

We summarize our results in Fig. 7 and plot the $\zeta$ potential [deduced from the measure of the charge current and Eq. [13)] as a function of the Debye screening factor in the wetting and non-wetting cases. In this figure the $\zeta$ potential is normalized by the bare surface potential $V_{0}$, obtained from the analytic PB expression [18], as shown e.g. in the inset of Fig. 2 The overall conclusion from Fig. 7 is that non-wettability strongly amplifies the electrokinetic effects: the ratio between the $\zeta$ potential and the surface potential is much larger in the hydrophobic case as compared to the hydrophilic case. More precisely, in the wetting case the $\zeta$ potential is fixed by the electric properties of the surface, and coincides with the electric potential at the "plane of shear", $\zeta \approx V\left(z_{\mathrm{s}}\right)$, as is usually assumed [18]. Indeed, the simulation points for $\zeta$ are compared to the $\mathrm{PB}$ estimate for the electric potential, $V\left(z_{s}\right)$, showing an overall very good correspondence. Conversely, the $\zeta$ potential in the non-wetting case is dominated by the slip effect and the immobile Stern layer is completely absent. The effect of such a modification of the hydrodynamic surface properties can be accounted for by considering the partial slip $\mathrm{BC}$ in the electrokinetic current $I_{\mathrm{e}}=\int \mathrm{d} S \rho_{\mathrm{e}}(z) v(z)$, with $\rho_{\mathrm{e}}(z)$ the charge density and $v(z)$ the velocity profile, characterized by a slip length $b$. Within linearized PB description (valid for $e V_{0} \ll k_{\mathrm{B}} T$ ), the result for the current $I_{\mathrm{e}}$, Eq. (13), may then be written $I_{\mathrm{e}}=\left(\varepsilon_{\mathrm{d}} V_{0} / \eta\right)(1+\kappa b) f_{0}$ [1, 23]. For the $\zeta$ potential in the non-wetting case, this amounts to:

$$
\zeta=V_{0}(1+\kappa b),
$$

with $V_{0}$ the bare potential of the surface. A detailed derivation of this effect is given in Appendix $\mathrm{A}$

For important potentials $\left(e V_{0} \gtrsim k_{\mathrm{B}} T\right)$, a non-linear $\mathrm{PB}$ counterpart of this expression can be obtained (see appendix $\mathrm{A}$ section $\mathrm{A3}$ :

$$
\zeta=V_{0}\left(1+\kappa_{\mathrm{eff}} b\right)
$$

where the effective Debye length $\kappa_{\text {eff }}^{-1}$ is defined by $\kappa_{\text {eff }}=-\partial_{n} V(0) / V_{0}$, and goes to $\kappa^{-1}$ in the linear limit. An equivalent expression has been recently discussed in a molecular hydrodynamics study of electro-osmosis in clays 24]. This expression is compared to simulation results in Fig. 7 showing again a very good agreement.

It is possible to retrieve the expression of the amplification ratio using a simple argument: the streaming current is given by $I_{\mathrm{e}} \sim Q \bar{v}, Q$ being the charge of the EDL and $\bar{v}$ the average velocity of the EDL, with $\bar{v}=\kappa^{-1} \dot{\gamma}$ in the absence of slip, whereas $\bar{v}=\left(\kappa^{-1}+b\right) \dot{\gamma}$ with a slip length $b$. The amplification ratio is therefore given by $\bar{v}_{\text {slip }} / \bar{v}_{\text {no-slip }}=1+\kappa b$.

\section{Electro-osmosis}

The streaming current simulations show that electrokinetic measurements do not probe electrostatic properties of the system only: when slippage occurs at the walls, the $\zeta$ potential is much larger that the bare surface potential $V_{0}$. This also means that the electrokinetic phenomena used to move liquids in microfluidic systems could be strongly amplified by hydrodynamic slippage. In order to illustrate this interesting outlook, we performed electroosmosis simulations, using the same numerical system. A uniform electric field $E_{x}$ applied in the channel induces a volume force inside the EDL, generating in fine a plug flow of the liquid. The standard description of this phenomenon predicts again a linear relationship between the electro-osmotic velocity and the applied field:

$$
v_{\mathrm{eo}}=-\left(\varepsilon_{\mathrm{d}} \zeta / \eta\right) E_{x} .
$$

In the simulation, we imposed an electric force $f_{x}=q e E_{x}$ to every ion (qe being the ion charge), and we measured the resulting velocity profile in the channel. We then used Eq. (16) to compute the corresponding $\zeta$ potential (the results presented were obtained for $E_{x}=1.0$ in Lennard-Jones units). As for the streaming current, linear response in the applied electric field was checked. 


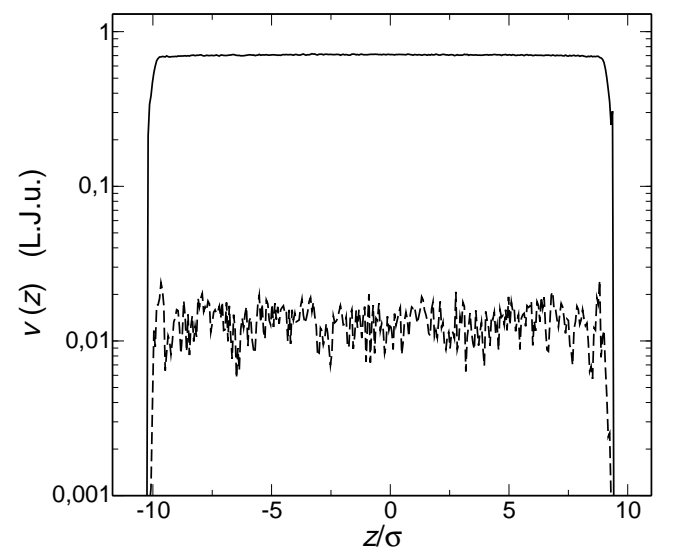

FIG. 8: Solvent velocity profile $v(z)$, averaged over the $x y$ directions, in a typical electro-osmosis simulation $\left(\rho_{\mathrm{s}} \sigma^{3}=0.06\right)$. An electric field $E_{x}$ is applied along the $x$ direction, leading to a plug velocity profile in the cell. The dashed line corresponds to the wetting case $\left(c_{F S}=1\right)$, whereas the solid line corresponds to the non-wetting case $\left(c_{F S}=0.5\right)$. The Debye screening factor is $\kappa \ell_{\mathrm{B}}=1.3$.

Typical velocity profiles are shown in figure 8 . For both wetting and non-wetting situations, we observe a plug flow, characteristic of electro-osmosis. Moreover, we note that the electro-osmotic velocity is considerably amplified - by almost two orders of magnitude - in the nonwetting case, all electric parameters being equal. The $\zeta$ potential, computed from the measured electro-osmotic velocity using equation (16), are in perfect agreement with those obtained in streaming current simulations.

It is possible to recover the slip prediction for $\zeta$ in the electro-osmosis case by stating that just outside the EDL, the viscous stress $\sigma_{\eta}$ compensates the electric forcing $\sigma_{\mathrm{e}}$ (integrated over the Debye layer). Writing $\sigma_{\eta}=\eta \partial_{z} v \sim$ $\eta v_{\mathrm{eo}} /\left(\kappa^{-1}+b\right)$ and $\sigma_{\mathrm{e}}=Q E_{x}=-\left(\varepsilon_{\mathrm{d}} \kappa V_{0}\right) E_{x}, Q=-\Sigma$ being the net surface charge of the EDL, one immediately finds :

$$
v_{\mathrm{eo}}=-\frac{\varepsilon_{\mathrm{d}} V_{0}(1+\kappa b)}{\eta} E_{x}=-\frac{\varepsilon_{\mathrm{d}} \zeta}{\eta} E_{x},
$$

with $\zeta=V_{0}(1+\kappa b)$. The amplification of the zeta potential thus originates in the reduction of the velocity gradient in the Debye layer by a factor $\kappa^{-1} /\left(\kappa^{-1}+b\right)$.

\section{Influence of electric parameters}

We finally investigate the influence of electric parameters on $\zeta$ potential. Fig. 9 presents a comparison between the measured $\zeta$ potential and the slip prediction (15), for various electric parameters (with added salt). The excellent correspondence between the two quantities confirm the robustness of the suggested picture, namely the $\zeta$ potential originates in the coupling between electrostatic properties of the ion cloud and the hydrodynamic behavior of the solvent, in the vicinity of charged surfaces.

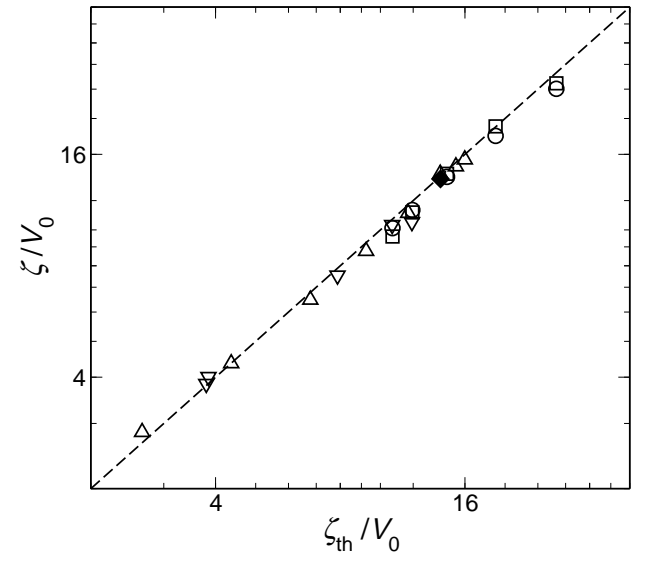

FIG. 9: Measured $\zeta$ potential versus slip prediction $\zeta_{\text {th }}$ for various situations: previous streaming current (o) and electroosmosis ( $\square$ ) simulations with fixed $\ell_{\mathrm{B}}=\sigma$ and $\Sigma=0.2 e / \sigma^{2}$; new streaming current simulations with: fixed $\ell_{\mathrm{B}}=\sigma$ and varying surface charge $\Sigma \sigma^{2} / e \in[0.1 ; 0.8](\triangle)$; larger Bjerrum length $\ell_{\mathrm{B}}=2 \sigma(\diamond)$; non-wetting solvent $\left(c_{F S}=0.5\right)$, various ions wetting properties and concentration $\left(c_{I S} \in[0.6 ; 1.0]\right.$ and $\left.\rho_{\mathrm{s}} \sigma^{3} \in[0.004 ; 0.14]\right)(\nabla) . \zeta$ potentials are normalized by the bare surface potential $V_{0}$ (see the discussion on the inset of Fig. 2). The dashed line represents $\zeta=\zeta_{\text {th }}$ as given By Eq. (15).

In the no-salt case, it is not possible to relate the streaming current to the zeta potential only because the electric potential is not fully screened over the fluid slab. However the simulations results are very well described by a simple model which takes into account the electric properties at a PB level and the hydrodynamic ones at a continuum level in the presence of slip (results not shown).

We now focus on the role of surface charge $\Sigma$. Figure [10] shows the evolution of the absolute $\zeta$ potential with surface charge. An important point is that it is possible to fully account for the simulation results with analytical expressions: in the wetting case, we simply used the value of the electric potential at the plane of shear $V\left(z_{\mathrm{s}}\right)$, together with the analytical fit for $z_{\mathrm{s}}(\Sigma)$, derived in section IV in the non-wetting case, we combined the formula (15), relating $\zeta$ to $b$ and electric parameters, with the analytical expression for $b(\Sigma)$, (12) derived in section IV Moreover, we used the $\mathrm{PB}$ expression for the evolution of electric parameters involved in $\zeta$ expression $\left(V(z), V_{0}, \kappa_{\text {eff }}\right)$ as a function of surface charge.

The non monotonous behaviour of $\zeta$ with $\Sigma$ is an interesting feature: while the bare surface potential $V_{0}$ increases continuously with $\Sigma$, the amplification factor related to the $\mathrm{HBC}$ - decreases, following an increase of electric friction $\left(1 / b^{\prime}\right)$. Those two conflicting effects result in a non-monotonous evolution, and the apparition of a maximum for $\zeta(\Sigma)$. This represents a particularly important signature of the intimate coupling between hydrodynamics and electrostatics in the definition of the $\zeta$ potential. We now discuss some consequences of these 


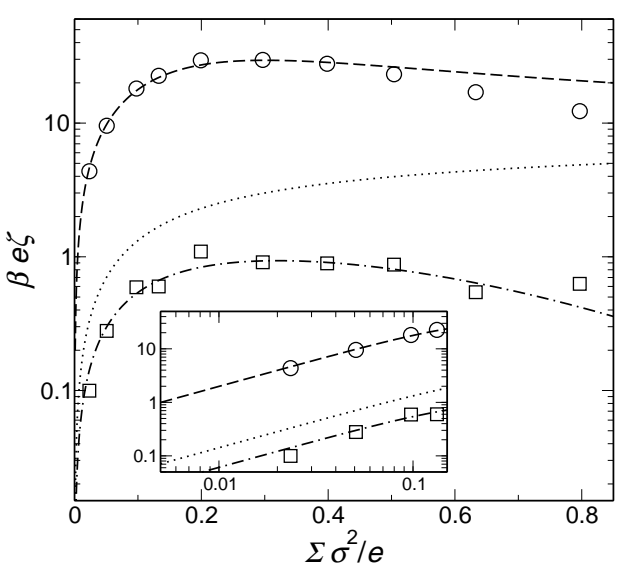

FIG. 10: Zeta potential as a function of surface charge $\Sigma$ $\left(\ell_{\mathrm{B}}=\sigma, \rho_{\mathrm{s}} \sigma^{3}=0.06\right)$. Symbols: MD results for the wetting $(\square)$ and the non-wetting (o) case. Dotted line: bare surface potential $V_{0}$, obtained from the PB prediction at a given $\kappa=$ $1.3 \sigma$ (see the discussion in the inset of Fig. 2). Dashed line: slip prediction using the analytical fit of $b$ variation with $\Sigma$ in Eq. (12) (see Fig. [5] and associated text). Dashed-dotted line: no-slip prediction using the analytical fit of $z_{\mathrm{s}}$ variation with $\Sigma$ (see Fig. [6 and associated text). The inset is a zoom in the small $\zeta$ small $\Sigma$ region.

results in the following section.

\section{DISCUSSION}

\section{A. $\zeta$ potential on hydrophobic surfaces : a slippage effect ?}

As we have shown above, the interplay between surface properties and electrokinetic phenomena may lead to $\zeta$ potential much higher than the real surface potential on hydrophobic surfaces, due in particular to the modification of surface hydrodrodynamic properties and the onset of liquid/solid slippage. In realistic experimental situations, the dynamical amplification ratio $1+\kappa b$ can take important values, even in the case of a moderate slip length : indeed, slip lengths are expected to range in the tens of nanometers range, as predicted theoretically [2] and evidenced experimentally [5, 8], while the Debye length $\kappa^{-1}$ is typically of a few nanometers in quite standard aqueous electrolyte solutions (from $3 \AA$ with a $1 \mathrm{M}$ salt, to $30 \mathrm{~nm}$ at $10^{-4} \mathrm{M}$ ). A factor of 10 between $\zeta$ and $V_{0}$ is therefore quite conceivable.

This analysis therefore provides an interesting scenario to interpret the observation of important $\zeta$ potentials on different hydrophobic surfaces [25, 26, 27, 28, 29]. Despite the expected weaker surface charge, the measured $\zeta$ potentials are typically of the same order of magnitude as those observed on hydrophilic surfaces [30]. This could in fact arise from small bare surface potentials and slip-induced amplification.
We note however that another interpretation is usually suggested: surface charge could be increased by preferential adsorption of anions $\left(\mathrm{OH}^{-}\right.$or $\left.\mathrm{Cl}^{-}\right)$at the wall. Recent AFM [31] and numerical 32] studies show that this phenomenon indeed contributes to a certain extent to increase surface charge.

Finally we mention a recent experimental work by Churaev et al. 23], who performed $\zeta$ potential measurements for aqueous $\mathrm{KCl}$ solutions in silanized quartz capillaries. When a non-ionic surfactant is added to the solution, the measured $\zeta$ potential decreases; this effect is attributed to the disappearance of hydrodynamic slippage at the walls, resulting from the surfactant adsorption. Using formula (14), the authors estimate the effective slip length (averaged over the surface of the channel) to lie between $5 \mathrm{~nm}$ and $8 \mathrm{~nm}$. This first experiment using electrokinetics to characterize the dynamical properties of the liquid at the interfaces is encouraging, but it seems that the results should be interpreted with care. Indeed, to apply Eq. (14), the authors assume that the adsorption of the surfactant only affects wetting properties of the surface (and therefore slip properties), electric properties being unaffected, which is difficult to assess.

To overcome those obstacles, comparative static (using SFA or AFM) and dynamic (with electrokinetics) measurements on the same surfaces would enable to test critically the relevance of the mechanisms suggested by this work. To the best of our knowledge, no such experiment has already been carried on.

\section{B. Zeta potential versus surface charge}

In light of the previous results, it may be possible to generate very large electrokinetic effects by using polarized hydrophobic surfaces 33]. However, we saw in sections IV and $\nabla$ that eventually, too high surface charges will enhance friction and therefore reduce the efficiency of hydrodynamic slippage. Consequently, there should be an optimal choice for the imposed surface charge, where the bare surface potential reaches its maximum value compatible with a small contribution of electric terms to the friction, therefore leaving the slip length almost unaffected (see Fig. 10).

To determine the largest achievable zeta potential according to our model, we now estimate the cross-over charge at which the electric friction equals the LJ friction, which gives $\Sigma_{\mathrm{c}} \sim 0.3 e / \sigma^{2}$. For $\sigma=0.5 \mathrm{~nm}$, this value corresponds roughly to $0.2 \mathrm{C} / \mathrm{m}^{2}$. Furthermore, using $\ell_{\mathrm{B}}=\kappa=\sigma$, we find for the corresponding bare surface potential $V_{0} \sim 80 \mathrm{mV}$ (and therefore $\left.\zeta \sim 10 V_{0} \sim 800 \mathrm{mV}\right)$.

This estimation for the optimal $\zeta$ potential is ten times larger than typical values which can be obtained on hydrophilic surfaces [30]. This should motivate experiments to test this prediction. We believe that the phenomena discussed provide interesting hints concerning the possibility to generate efficient electro-osmotic flows, with 
direct applications in microfluidics.

\section{Curvature effects}

Our prediction for the dynamical amplification ratio, $1+\kappa b$ can take arbitrarily large values as the slip length $b$ goes to infinity (corresponding to a vanishing shear boundary condition at the interface). This prediction corresponds however to a planar surface and we show in this paragraph that curvature effects actually lead to a saturation of the amplification ratio for the zeta potential.

To demonstrate this effect, we shall generalize the calculation of electrophoretic mobility of a sphere initiated by Smoluchowski 34 in 1921, to take into account the partial slip BC at the sphere surface.

Let us consider a dielectric sphere, with radius $a$, immersed in an electrolyte solution and submitted to an external electric field $\boldsymbol{E}_{\infty}$. We assume that the width of the EDL is small compared to the size of the sphere $(\kappa a \gg 1)$, and that the sphere permittivity is small compared to the one of the liquid [46]. Finally we consider that the external electric field does not modify the ions distribution inside the EDL [47]. We then solve the equations of the problem separately inside and outside the EDL. We note $\mathcal{S}^{+}$the sphere separating the EDL and the exterior.

Since the sphere permittivity is very low, the normal component of the electric field vanishes just outside the EDL. Inside the EDL, the problem is reduced to an electro-osmosis one: neglecting the $1 / r^{2}$ terms in the Laplacian of the velocity field, we recover literally the equations of the plane case, simply replacing $v_{t}$ with $v_{\theta}$ and $n$ with $r$ 48]. Thus we can immediately write:

$$
\left.\boldsymbol{v}_{\|}\right|_{\mathcal{S}^{+}}=-\left.\frac{\varepsilon_{\mathrm{d}} \zeta}{\eta} \boldsymbol{E}_{\|}\right|_{\mathcal{S}^{+}} .
$$

Finally, for an irrotational flow, the potential of velocities obeys Laplace equation in the liquid outside the EDL, just as the electric potential. As the BCs at infinity and on $S^{+}$are identical, we can identify both fields at the constant $\varepsilon_{\mathrm{d}} \zeta / \eta$. We directly deduce that $\boldsymbol{v}_{\mathrm{e}}=\left(\varepsilon_{\mathrm{d}} \zeta / \eta\right) \boldsymbol{E}_{\infty}$.

Nevertheless, the partial slip BC has to be modified to take into account the sphere curvature [35]: indeed, this condition accounts for the equality of friction $\lambda v_{\theta}$ and viscous $\sigma_{r \theta}$ tangential stresses, but in spherical coordinates the expression of the stress tensor at a radius $a$ includes a curvature term: $\sigma_{r \theta}=\eta\left(\partial_{r} v_{\theta}-v_{\theta} / a\right)$. We obtain a generalized slip condition at a surface with curvature $a$ :

$$
\frac{\partial v_{\theta}}{\partial r}=v_{\theta}\left(\frac{1}{b_{0}}+\frac{1}{a}\right),
$$

where $b_{0}=\eta / \lambda$ is the intrinsic slip length on a plane surface; we define the slip length $b_{\mathrm{c}}$ on a curved surface by:

$$
\frac{1}{b_{\mathrm{c}}}=\frac{1}{b_{0}}+\frac{1}{a}
$$

This equation shows that slippage is limited by the smallest of $b_{0}$ and $a$ and curvature leads to a saturation of the effective slip length as $b_{0} \rightarrow \infty$. Coming back to the calculation of the zeta potential, the problem is equivalent to that of a planar surface with slip length $b_{c}$. We therefore simply deduce from Eq. (14) the effective $\zeta$ potential:

$$
\zeta=V_{0}\left(1+\kappa b_{\mathrm{c}}\right)
$$

The zeta potential therefore depends on the ratio $a / b_{0}$, with the limiting values : $\zeta_{0}=V_{0}\left(1+\kappa b_{0}\right)$ for $b_{0} \ll a$, independent of the radius of the particle; and $\zeta_{c}=V_{0}(1+$ $\kappa a)$ in the opposite limit $b_{0} \gg a$.

\section{CONCLUSION}

The liquid properties close to an interface, embodied in the so-called Hydrodynamic Boundary Condition is intimately related to the friction properties of the liquid at the solid surface. In this paper we investigated extensively the electric contribution to liquid/solid friction, and how it affects the slip properties of liquids in the presence of charged walls. Various electric parameters were considered, and we provided a simple but efficient model of electric friction, validated numerically thanks to Molecular Dynamics simulations. This model describes the slip length $b$ dependence with the surface charge $\Sigma$ and the Bjerrum length $\ell_{\mathrm{B}}$ of the solvent. Moreover, it explains the weak dependence of $b$ with the salt concentration measured in the simulations.

We then addressed the consequences of such a hydrodynamic slippage on electrokinetic phenomena, through the coupling between hydrodynamics and electric charge within the Electric Double Layer. In this work, we extended a previous study [13], by considering various electric parameters and electrokinetic configurations. We confirmed the robustness of the suggested picture, namely the widely used "zeta potential" - characterizing the amplitude of electrokinetic effects - is not only a signature of electrostatic interfacial features, but is also intrinsically related to the dynamics of the solvent at the solid surface, providing new perspectives to control this quantity. A similar conclusion was reached in recent work [24]. In particular, we showed the existence of strongly amplified electro-osmotic effects on hydrophobic surfaces through the induced slippage, in quantitative agreement with previous streaming current simulations. In the slipping case, we discussed the non-trivial role of the surface charge $\Sigma$ on the $\zeta$ potential: in addition to its direct influence on the surface potential $V_{0}$, it enhances friction and therefore reduces the efficiency of hydrodynamic slippage. These conflicting effects lead to a non-monotonous variation of $\zeta$ with $\Sigma$, which represents an important signature of the coupling mechanisms suggested here. Besides, the simulation results were shown to be in excellent agreement with predictions taking into account the slippage of the fluid at the solid surface. The amplification 


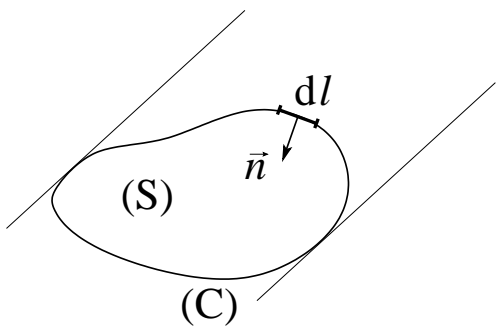

FIG. 11: Illustration of the channel used for $\zeta$ calculation.

effect is accordingly controlled by the ratio between the slip length (of the fluid at the solid surface), and the Debye length.

Furthermore, practical consequences of our work have been discussed, reconsidering existing experimental data of $\zeta$ potentials on hydrophobic surfaces, and suggesting the possibility to generate strongly enhanced electroosmotic and electrophoretic effects in microchannels, using in particular polarized hydrophobic surfaces. If confirmed, this feature would provide various interesting applications for microfluidic devices.

Finally, this work suggests the possibility to use the zeta potential as a new observable for the characterization of interfacial hydrodynamics, through the coupling at small scales of electrostatics and hydrodynamics. In a following step, we plan to refine the model, with a more realistic description of solvent and ions $(\mathrm{SPC} / \mathrm{E}$ model of water, ions with different sizes and wetting properties, etc.), in order to relate in a more quantitative way electrokinetics measurements and slippage properties.

\section{Acknowledgments}

The authors wish to thank Armand Ajdari for fruitful discussions.

\section{APPENDIX A: ZETA POTENTIAL AND SLIP}

We detail here how the standard calculation of $\zeta$ potential [18] is modified when a partial slip BC applies at the walls, in both streaming current and electro-osmosis situations.

\section{Streaming current}

To calculate the streaming current expression, we consider a channel of arbitrary section (Fig. 11). We assume that the Debye length $\kappa^{-1}$ is small compared to the typical size of the channel $R$ and to the local inverse curvature of the channel $r$ everywhere on the contour $\mathcal{C}$. We can therefore consider that the channel surface is flat at the EDL scale.
The elementary contribution to streaming current due to the displacement of the EDL past a channel slice of width $\mathrm{d} l$ is:

$$
\mathrm{d} I_{\mathrm{e}}=\mathrm{d} l \int_{0}^{\infty} \rho_{\mathrm{e}}(n) v(n) \mathrm{d} n
$$

where $\infty \gg \kappa^{-1}$ and $n$ is the coordinate normal to the surface. We then substitute $\rho_{\mathrm{e}}$ using Poisson equation:

$$
\mathrm{d} I_{\mathrm{e}}=-\varepsilon_{\mathrm{d}} \mathrm{d} l \int_{0}^{\infty} \frac{\partial^{2} V}{\partial n^{2}} v(n) \mathrm{d} n .
$$

To evaluate this integral, we must introduce the boundary conditions for the velocity $v$ and the potential $V$ at the wall $(z=0)$ and far from the wall $(z=\infty)$. The velocity follows a partial slip BC: $v(0)=b \partial_{n} v$ (we assume that $b \ll r$, so that curvature effects can be neglected [35]); we note $V_{0}=V(0)$ the electric potential at the wall. Far from the wall, the potential is constant $\left(\partial_{n} V=0\right)$, conveniently fixed at $V(\infty)=0$. We can now integrate by parts the expression in A2):

$$
\begin{aligned}
& \mathrm{d} I_{\mathrm{e}}=-\varepsilon_{\mathrm{d}} \mathrm{d} l\left\{\left[\frac{\partial V}{\partial n} v\right]_{0}^{\infty}-\int_{0}^{\infty} \frac{\partial V}{\partial n} \frac{\partial v}{\partial n} \mathrm{~d} n\right\} \\
& =-\varepsilon_{\mathrm{d}} \mathrm{d} l\{-\underbrace{\left.\frac{\partial V}{\partial n}\right|_{0} v(0)}_{\sim \kappa V_{0} \bar{v}}-\underbrace{\left[V \frac{\partial v}{\partial n}\right]_{0}^{\infty}}_{\sim \kappa V_{0} \bar{v}}+\underbrace{\int_{0}^{\infty} \frac{\partial^{2} \vartheta}{\partial n^{2}} \mathrm{~d} n}_{\sim \kappa V_{0} \bar{v} \mathcal{O}\left(\kappa^{-1} / R\right)}\} \\
& =-\varepsilon_{\mathrm{d}} \mathrm{d} l\left\{-\left.\frac{\partial V}{\partial n}\right|_{0} \times\left. b \frac{\partial v}{\partial n}\right|_{0}+\left.V_{0} \frac{\partial v}{\partial n}\right|_{0}\right\} \\
& =-\varepsilon_{\mathrm{d}} \mathrm{d} l \times\left. V_{0} \frac{\partial v}{\partial n}\right|_{0}\left\{1+b \frac{-\partial_{n} V(0)}{V_{0}}\right\} \text {. }
\end{aligned}
$$

We then integrate this expression on the contour of the channel:

$$
I_{\mathrm{e}}=-\varepsilon_{\mathrm{d}} V_{0}\left(1+b \frac{-\partial_{n} V(0)}{V_{0}}\right) \times \oint_{\mathcal{C}} \frac{\partial v}{\partial n} \mathrm{~d} l .
$$

The second Green theorem [36] enables us to transform this expression:

$$
I_{\mathrm{e}}=-\varepsilon_{\mathrm{d}} V_{0}\left(1+b \frac{-\partial_{n} V(0)}{V_{0}}\right) \times \iint_{\mathcal{S}} \Delta v \mathrm{~d} S .
$$

The Laplacian of velocity is directly related to forcing through Stokes equation: $\Delta v=(1 / \eta)(-\nabla p)=$ Cst. We finally obtain:

$$
I_{\mathrm{e}}=-\frac{\varepsilon_{\mathrm{d}} V_{0}\left(1+\kappa_{\mathrm{eff}} b\right)}{\eta} \times \mathcal{A} \times(-\nabla p),
$$

where the effective Debye length $\kappa_{\text {eff }}^{-1}$ is defined by $\kappa_{\text {eff }}=$ $-\partial_{n} V(0) / V_{0}$, and goes to $\kappa^{-1}$ in the linear limit $e V_{0} \ll$ $k_{\mathrm{B}} T(\mathcal{A}$ is the area of the channel section $\mathcal{S})$. The $\zeta$ potential, derived from streaming current measurements, is therefore related to the bare surface potential by a dynamical amplification factor arising from slip:

$$
\zeta=V_{0}\left(1+\kappa_{\mathrm{eff}} b\right)
$$

The effective length $1 / \kappa_{\text {eff }}$ is discussed in section A3 


\section{Electro-osmosis}

To calculate the electro-osmotic velocity on the same system, we solve Stokes equation thanks to the BCs introduced in the preceding sub-section:

$$
-\eta \frac{\partial^{2} v}{\partial n^{2}}=\rho_{\mathrm{e}} E_{\mathrm{t}} .
$$

We replace $\rho_{e}$ by its expression derived from Poisson equation:

$$
\eta \frac{\partial^{2} v}{\partial n^{2}}=\varepsilon_{\mathrm{d}} E_{\mathrm{t}} \frac{\partial^{2} V}{\partial n^{2}} .
$$

This equation is integrated, assuming $\partial_{n} V$ and $\partial_{n} v$ both vanish far from the wall:

$$
\eta \frac{\partial v}{\partial n}=\varepsilon_{\mathrm{d}} E_{\mathrm{t}} \frac{\partial V}{\partial n} .
$$

From this equation, we deduce that $v(0)=b \partial_{n} v(0)=$ $b\left(\varepsilon_{\mathrm{d}} E_{\mathrm{t}} / \eta\right) \partial_{n} V(0)$. A second integration between the wall - where $V=V_{0}$ and $v(0)=b \partial_{n} v(0)$ - and a plane located outside the EDL - where $V=0$ and $v=\mathrm{Cst}=v_{\text {eo }}$ - leads to:

$$
\begin{aligned}
v_{\mathrm{eo}}-v(0) & =-\frac{\varepsilon_{\mathrm{d}} E_{\mathrm{t}}}{\eta}\left(V_{0}-0\right) \\
v_{\mathrm{eo}} & =-\frac{\varepsilon_{\mathrm{d}} E_{\mathrm{t}}}{\eta}\left(V_{0}-\left.b \frac{\partial V}{\partial n}\right|_{0}\right),
\end{aligned}
$$

from which we extract the electro-osmotic velocity:

$$
v_{\mathrm{eo}}=-\frac{\varepsilon_{\mathrm{d}} \zeta}{\eta} \times E_{\mathrm{t}},
$$

with the $\zeta$ potential:

$$
\zeta=V_{0}\left(1+\kappa_{\mathrm{eff}} b\right)
$$

The fact that we get the same $\zeta$ potential for both phenomena complies with the Onsager reciprocity theorem.

\section{The effective Debye length}

In section $\mathrm{VB}$ we introduced the length scale $1 / \kappa_{\text {eff }}=$ $-V_{0} / \partial_{n} V(0)$ that allows to relate the surface potential $V_{0}$, the slip length $b$ and the effective $\zeta$ potential [see e.g. Eq. (15)]. In the weak overlap regime where the double layer is not affected by the opposite boundary, one may use the analytical solution of PB theory in the planar geometry 37] to obtain

$$
\kappa / \kappa_{\mathrm{eff}}=\operatorname{argth}(\gamma) \frac{1-\gamma^{2}}{\gamma}
$$

where $\gamma=-\kappa \ell_{\mathrm{GC}}+\sqrt{\left(\kappa \ell_{\mathrm{GC}}\right)^{2}+1}$ and $\ell_{\mathrm{GC}}$ denotes the Gouy length $\ell_{\mathrm{GC}}=\left(2 \pi \ell_{B} \Sigma\right)^{-1}$. In the weak coupling regime (low $\Sigma$ where $\gamma$ vanishes), we find $\kappa_{\text {eff }} \simeq \kappa$ while conversely, for high charges, $\kappa / \kappa_{\text {eff }} \sim-\kappa \ell_{\mathrm{GC}} \log \left(\kappa \ell_{\mathrm{GC}}\right)$ which decreases as $\log (\Sigma) / \Sigma$.

The effective length $1 / \kappa_{\text {eff }}$ is reminiscent of a related quantity $l_{G W}=-\partial_{n} V(0) / \partial_{n}^{2} V(0)$ introduced some time ago as a scaling length for charged interfaces [38]. Upon increasing the surface charge, $l_{G W}$ crosses over from the Debye length at low $\Sigma$ (as $\left.1 / \kappa_{\text {eff }}\right)$ to the Gouy length. It therefore decreases as $1 / \Sigma$ for large interfacial charges, whereas $1 / \kappa_{\text {eff }}$ is slightly larger $[\propto \log (\Sigma) / \Sigma]$.

Finally, it appears that the increase of $\kappa_{\text {eff }}$ at large $\Sigma$ due to non linear effects is overruled by the decrease of the slipping length $\left(b \propto 1 / \Sigma^{2}\right.$, see section IVB $)$ : increasing $\Sigma$ decreases the slip-driven amplification factor $1+\kappa_{\text {eff }} b$, see Fig 10.

\section{APPENDIX B: WETTING PROPERTIES OF IONS}

To obtain the present results, we considered a very simple model with identical wetting properties for all liquid atoms, including ions. This specific choice of LJ parameters aims at separating electric properties at the interface from the dynamical slipping behavior of the liquid. To separate these effects, ions were therefore supposed to interact with the wall in the same way as the solvent, so as to keep the interfacial friction unaffected. Moreover, no effect of salt on slip length has been reported experimentally in the literature, therefore suggesting that no specific behavior of the ions as compared to the solvent occurs at the solid surface. In other words, solventsurface and ion-surface interactions are expected to be rather similar, and not very asymmetric. However, so as to explore the influence of our specific choice of LJ interactions on the solvent dynamics, we performed complementary simulations, exploring various solvent-surface $\left(c_{F S}\right)$ and ion-surface $\left(c_{I S}\right)$ interactions, in the parameter window $c_{F S}, c_{I S} \in[0.5 ; 1]$ (with different values for $c_{F S}$ and $c_{I S}$ ), for different salt concentrations. We calculated both equilibrium and dynamical quantities for these parameters.

Let us first focus on the equilibrium properties of the EDL. For moderately asymmetric situations $\left(\delta c_{I S} \lesssim\right.$ 0.2 ), the modified PB approach (Sec. III) remains valid. Indeed, as can be seen on Fig. [12 the standard PB potential (without structuration) describes quite well the electric potential, computed from the simulation by double-integration of the charge density profile $\rho_{\mathrm{e}}(z)=$ $e\left(\rho_{+}(z)-\rho_{-}(z)\right)$. Nevertheless, for strongly asymmetric situations - typically with a "non-wetting" solvent $\left(c_{F S}=0.5\right)$ and "wetting" ions $\left(c_{I S}=1\right)-$, we observe specific ions-wall interactions, and the modified PB model fails to describe the electric potential at the interface (see Fig. 12). To conclude on the static properties, the modified PB approach, even if it fails to describe specific ions-solid interactions, provides an accurate description for moderately asymmetric parameters, which we think are representative of experimental situations. 


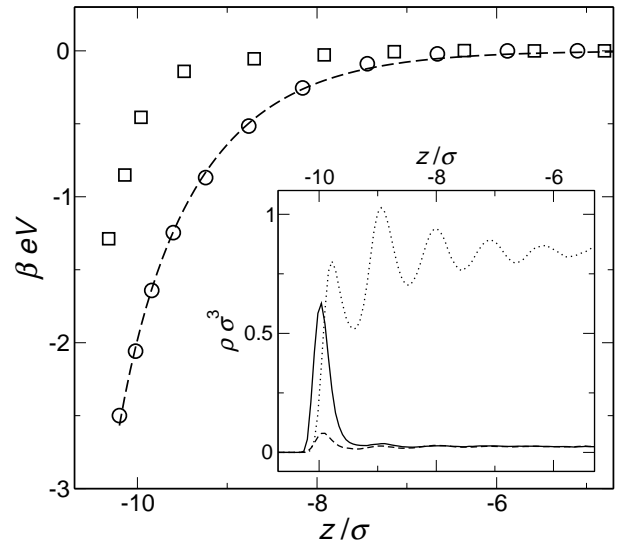

FIG. 12: Electrostatic potential for a non-wetting solvent $\left(c_{F S}=0.5\right)$ and ions with different wetting properties $\left(\rho_{\mathrm{s}} \sigma^{3}=\right.$ 0.07). Dashed line: bare PB prediction; Symbols: MD results for $c_{I S}=0.6(\circ)$ and $c_{I S}=1(\square)$. Inset: density profiles in the strongly asymmetric case $\left(c_{F S}=0.5\right.$ and $\left.c_{I S}=1\right)$ : solvent $(\cdots)$, counter-ions $(-)$ and co-ions $(--)$.

As far as dynamical properties are concerned, all the situations considered confirm the robustness of the picture put forward in the main text. On Fig. 9] we compare the $\zeta$ potentials measured from the simulations with the slip prediction (15). For moderately asymmetric situations, we used the PB potential as an input, whereas for strongly asymmetric ones we used the "exact" surface potential, extracted from the simulations. These quantities correspond to each other perfectly. Thus these complementary results confirm that the message emerging from the simpler case of equal LJ interactions is not modified when exploring a larger parameter space: namely the zeta potential is a signature of the coupling between the electrostatics and the fluid dynamical properties at the surface.

\section{APPENDIX C: DENSITY FUNCTIONAL THEORY AND THE MODIFIED POISSON-BOLTZMANN EQUILIBRIUM}

For arbitrary solvent and microionic density profiles $\left[\right.$ resp $\rho_{f}(\boldsymbol{r})$ and $\left.\rho_{ \pm}(\boldsymbol{r})\right]$, the total free energy of our confined system may be written as the functional

$$
\begin{aligned}
\beta \mathcal{F}\left\{\rho_{f}, \rho_{ \pm}\right\} & =\sum_{i \in f,+,-} \int_{\mathcal{V}} \rho_{i}(\boldsymbol{r})\left[\log \left(\Lambda_{i}^{3} \rho_{i}(\boldsymbol{r})\right)-1\right] d \boldsymbol{r} \\
& +\beta \mathcal{F}_{\text {excess }}^{\mathrm{LJ}}\left\{\rho_{\mathrm{tot}}\right\}+\beta \mathcal{F}^{\mathrm{Coul}}\left\{\rho_{+}, \rho_{-}\right\},(\mathrm{C} 1
\end{aligned}
$$

where $\mathcal{V}$ denotes the total volume and the $\Lambda_{i}$ are irrelevant length scales. The first term on the rhs acounts for the ideal gas entropy while the excess part is included in $\mathcal{F}_{\text {excess }}^{\mathrm{LJ}}$. This a priori unknown functional depends on the total density only

$$
\rho_{\text {tot }}=\rho_{f}+\rho_{+}+\rho_{-} .
$$

This is a consequence of treating solvent and microions on equal footings, not only in their mutual interactions, but also in their interaction with wall atoms. Finally, the last term on the rhs of Eq. (C1) denotes the Coulombic contribution to the total free energy. Unlike its Lennard-Jones counterpart, treated exactly (but formally), $\mathcal{F}^{\text {Coul }}\left\{\rho_{+}, \rho_{-}\right\}$is approximated, in a mean-field spirit (see e.g. [17]), by

$$
\beta \mathcal{F}^{\text {Coul }}\left\{\rho_{+}, \rho_{-}\right\}=\frac{1}{2} \int_{\mathcal{V} \times \mathcal{V}} \rho_{e}(\boldsymbol{r}) G\left(\boldsymbol{r}, \boldsymbol{r}^{\prime}\right) \rho_{e}\left(\boldsymbol{r}^{\prime}\right) d \boldsymbol{r} d \boldsymbol{r}^{\prime}
$$

where $G\left(\boldsymbol{r}, \boldsymbol{r}^{\prime}\right)$ denotes the Green's function inverting the Laplacian in the geometry under study and $\rho_{e}(\boldsymbol{r})=$ $e\left[\rho_{+}(\boldsymbol{r})-\rho_{-}(\boldsymbol{r})\right]$ is the local charge density, outside the confining walls.

Minimizing the total free energy $\mathcal{F}$ with respect to solvent density leads to

$$
\log \left[\Lambda_{s}^{3} \rho_{s}(\boldsymbol{r})\right]+\frac{\delta \beta \mathcal{F}_{\text {excess }}^{\mathrm{LJ}}}{\delta \rho_{\mathrm{tot}}(\boldsymbol{r})}=\mathrm{cst}
$$

both in the canonical ensemble where the total number of solvent molecules is fixed, or in a grand canonical description where the chemical potential is given. Here, explicit use was made of Eq. (C2). On the other hand, minimization with respect to microionic densities yields

$\log \left[\Lambda_{ \pm}^{3} \rho_{ \pm}(\boldsymbol{r})\right]+\frac{\delta \beta \mathcal{F}_{\mathrm{excess}}^{\mathrm{LJ}}}{\delta \rho_{\mathrm{tot}}(\boldsymbol{r})} \pm \beta e \int_{\mathcal{V}} \rho_{e}\left(\boldsymbol{r}^{\prime}\right) G\left(\boldsymbol{r}, \boldsymbol{r}^{\prime}\right) d \boldsymbol{r}^{\prime}=\mathrm{cst}^{\prime}$

Inserting Eq. C4 into (C5) and realizing that the electric potential reads

$$
\beta e V(\boldsymbol{r})=\int_{\mathcal{V}} \rho_{e}\left(\boldsymbol{r}^{\prime}\right) G\left(\boldsymbol{r}, \boldsymbol{r}^{\prime}\right) d \boldsymbol{r}^{\prime}
$$

we obtain

$$
\rho_{ \pm}(\boldsymbol{r}) \propto \rho_{s}(\boldsymbol{r}) e^{\mp \beta e V(\boldsymbol{r})} .
$$

This is precisely the form obtained in Eq. (41) on more heuristic grounds. Upon neglecting the LJ excess free energy, one recovers the standard Poisson-Boltzmann relation.

The fact that the Lennard-Jones free energy functional only depends on the total density $\rho_{\text {tot }}$ given by $\mathrm{C2}$ plays a pivotal role in the derivation of (C7) and results from the symmetrical role played by ions and solvent molecules as far as non ionic interactions are concerned. Whenever the wetting properties of the ions differ from those of the solvent, $\mathcal{F}_{\text {excess }}^{\mathrm{LJ}}$ no longer depends on $\rho_{\text {tot }}$ but in general separately on $\rho_{s}, \rho_{+}$and $\rho_{-}$. A similar remark applies if the non Coulombic solvent-solvent interaction differs from solvent-ion and ion-ion interactions. In those situations, Eq. (C7) does not hold, see e.g. Appendix B While analytical progress might be possible, we did not attempt a density functional description in these cases.

A criterion for the validity of the modified PoissonBoltzmann description (C7). Equation (C1) assumes a 
mean field factorization of the Coulombic energy, which discards microionic correlations. The importance of these correlations is quantified by the plasma parameter $\Gamma=$ $z^{2} \ell_{B} / d_{i i}$ where $d_{i i}$ denotes the typical distance between counterions of valency $z$ in the electric double layer [39]. This distance is most conveniently estimated assuming that the counterions form a Wigner crystal at the planar interface, which gives $d_{i i}^{2} \Sigma=z e$. We consequently obtain the following criterion for the validity of the mean-field assumption underlying the modified Poisson-Boltzmann picture :

$$
\Gamma=z^{3 / 2}\left(\Sigma \ell_{B}^{2} / e\right)^{1 / 2}<1 .
$$

A priori, the threshold value of $\Gamma$ is expected to be of order 1 , and a survey of the literature indicates that it is very close to unity, hence Eq. (C8) (see 39, 40] and references therein). At fixed $\ell_{B}=\sigma$, (C8) implies for monovalent microions $\Sigma \sigma^{2} / e<1$, which is always fulfilled in the situations investigated in the present study. We emphasize here that the short distance repulsion of the Lennard-Jones potential used ensures that $d_{i i}>\sigma$, so that one always has $\Gamma=\ell_{B} / d_{i i}<1$ for $\ell_{B}=\sigma$ (and more generally for $\ell_{B}<\sigma$ ). On the other hand, considering $\Sigma=0.2 e / \sigma^{2}$, (C8) translates, again for $z=1$, into $\ell_{B}<2.2 \sigma$. We thus expect here deviations from the modified $\mathrm{PB}$ equilibrium for $\ell_{B}>2.2 \sigma$. This point is discussed at the end of section
[1] H. Stone, A. Stroock, and A. Ajdari, Ann. Rev. Flu. Mech. 36, 381 (2004).

[2] J.-L. Barrat and L. Bocquet, Phys. Rev. Lett. 82, 4671 (1999).

[3] P. Joseph and P. Tabeling, Phys. Rev. E 71, 035303 (2005).

[4] T. Schmatko, H. Hervet, and L. Léger, Phys. Rev. Lett. 94, 244501 (2005).

[5] C. Cottin-Bizonne, B. Cross, A. Steinberger, and E. Charlaix, Phys. Rev. Lett. 94, 056102 (2005).

[6] O. I. Vinogradova and G. E. Yakubov, Phys. Rev. E 73, 045302 (2006).

[7] E. Lauga, M. P. Brenner, and H. A. Stone, in Handbook of Experimental Fluid Dynamics, edited by C. T. J. Foss and A. Yarin (Springer, 2005), chap. 15, to appear, cond-mat/0501557.

[8] L. Joly, C. Ybert, and L. Bocquet, Phys. Rev. Lett. 96, 046101 (2006).

[9] S. Granick, Y. Zhu, and L. Hyunjung, Nature Mat. 2, 221 (2003).

[10] Y. W. Kim and R. R. Netz, Europhys. Lett. 72, 837 (2005).

[11] Y. W. Kim and R. R. Netz, J. Chem. Phys. 124, 114709 (2006).

[12] R. R. Netz, Curr. Op. Coll. Int. Sci. 9, 192 (2004).

[13] L. Joly, C. Ybert, E. Trizac, and L. Bocquet, Phys. Rev. Lett. 93, 257805 (2004).

[14] R. Qiao and N. R. Aluru, Phys. Rev. Lett. 92, 198301 (2004).

[15] J. Lyklema, Fondamentals of interface and colloid science, vol. 2 (Academic Press, London, 1995).

[16] D. Andelman, in Membranes: Their Structure and Conformations, edited by R. Lipowsky and E. Sackmann (Elsevier, 1999).

[17] E. Trizac, Phys. Rev. E 62, R1465 (2000).

[18] R. J. Hunter, Zeta potential in colloid science (Academic Press, London, 1981).

[19] J.-L. Barrat and L. Bocquet, Faraday Discuss. 112, 119 (1999).

[20] L. Bocquet and J.-L. Barrat, Phys. Rev. E 49, 3079 (1994).

[21] M. Bazant and T. Squires, Phys. Rev. Lett. 92, 066101 (2004).
[22] R. R. Netz, Phys. Rev. Lett. 91, 138101 (2003).

[23] N. V. Churaev, J. Ralston, I. P. Sergeeva, and V. D. Sobolev, Adv. Coll. Int. Sci. 96, 265 (2002).

[24] J. F. Dufrêche, V. Marry, N. Malikova, and P. Turq, J. Mol. Liq. 118, 145 (2005).

[25] E. Chibowski and A. Waksmundzki, J. Colloid Interface Sci. 66, 213 (1978).

[26] J. Laskowski and J. A. Kitchener, J. Colloid Interface Sci. 29, 670 (1969).

[27] H. C. Parreira and J. H. Schulman, Advan. Chem. Ser. 33, 160 (1961).

[28] F. Ozon, J.-M. di Meglio, and J.-F. Joanny, Eur. Phys. J. E 8, 321 (2002).

[29] R. Schweiss, P. B. Welzel, C. Werner, and W. Knoll, Langmuir 17, 4304 (2001).

[30] P. J. Scales, F. Grieser, T. W. Healy, L. R. White, and D. Chan, Langmuir 8, 965 (1992).

[31] K. Hu and A. J. Bard, Langmuir 13, 5114 (1997).

[32] H. J. Kreuzer, R. L. C. Wang, and M. Grunze, J. Am. Chem. Soc. 125, 8384 (2003).

[33] R. Schasfoort, S. Schlautmann, J. Hendrikse, and A. van den Berg, Science 286, 942 (1999).

[34] M. von Smoluchowski, Handbuch der Electrizitt und des Magnetismus, vol. II (Graetz, 1921).

[35] D. Einzel, P. Panzer, and M. Liu, Phys. Rev. Lett. 64, 2269 (1990).

[36] I. S. Gradshteyn and I. M. Ryzhik, Table of integrals, series, and products (Academic press, 2000), sixth ed.

[37] R. J. Hunter, Foundations of colloid science (Oxford Univ. Press, Oxford, 2001), 2nd ed.

[38] G. Weisbuch and M. Guéron, J. Physique 44, 251 (1983).

[39] Y. Levin, Rep. Prog. Phys. 65, 1577 (2002).

[40] Y. Levin, E. Trizac, and L. Bocquet, J. Phys.: Condens. Matter 15, S3523 (2003).

[41] S. J. Plimpton, J. Comp. Phys. 117, 1 (1995).

[42] R. W. O'Brien and L. White, J. Chem. Soc. Faraday Trans. 2 74, 1607 (1978).

[43] R. W. O'Brien, J. Colloid Interface Sci. 92, 204 (1983).

[44] We have used the MD code LAMmPS 2001, by S. J. Plimpton 41], available at http://www.cs.sandia.gov/ sjplimp/lammps.html

[45] A possible reason is that for such high charges, the pure electric contribution $F_{\mathrm{ES}}^{2}$ cannot be neglected which leads 
to a decrease of $b$, as observed in Fig. [5]

[46] This hypothesis is reasonable for most of the particles in water, but is not necessary: O'Brien and White have shown that the mobility is independent of the particle permittivity [42] (using a no-slip boundary condition).
[47] This hypothesis can be relaxed: O'Brien [43] gave the complete solution (with a no-slip boundary condition).

[48] This approximation corresponds to neglecting terms of order $\mathcal{O}\left(1 /(\kappa a)^{2}\right.$. 